\title{
Outcomes of patients with End Stage Kidney Disease on dialysis with COVID-19. From PCR to Antibody.
}

Wasim Ahmed ( $\square$ wasahmed@seha.ae)

SEHA Kidney Care, Abu Dhabi, UAE

Ali Abdul Kareem Al Obaidli

SEHA Kidney Care, Abu Dhabi, UAE

Princy Joseph

SEHA Kidney Care, Abu Dhabi, UAE

Edward Smith

Univeristy of Melbourne

Ayaz Ahmad Khan

SEHA Kidney Care, Abu Dhabi, UAE

Siddiq Anwar

SEHA Kidney Care, Abu Dhabi, UAE

Thangavelu Chandrasekar

SEHA Kidney Care, Abu Dhabi, UAE

Ayman Kamal Al Madani

SEHA Kidney Care, Abu Dhabi, UAE

Hormazdiar Dara Dastoor

SEHA Kidney Care, Abu Dhabi, UAE

Imran Zahid

Sheikh Shakhbout Medical City, UAE

Freddie Agbayani Costales

SEHA Kidney Care, Abu Dhabi, UAE

Yousef Abdul Rahim Boobes

Tawam Hospital, Al Ain, UAE.

Fatima Al Kindi

Tawam Hospital, Al Ain, UAE.

Salah Eldin Khalil Issa

Madinat Zayed Hospital, UAE.

Mohammed Hassan Hassan

6. Sheikh Khalifa Medical City, UAE.

Abraham George 
Tawam Hospital, Al Ain, UAE.

Stephen G Holt

SEHA Kidney Care, Abu Dhabi, UAE

\section{Research Article}

Keywords: COVID-19, End stage Kidney disease, dialysis, IgG antibody, mortality, screening

Posted Date: October 2nd, 2020

DOI: https://doi.org/10.21203/rs.3.rs-85167/v1

License: (c) (1) This work is licensed under a Creative Commons Attribution 4.0 International License. Read Full License 


\section{Abstract}

Background: End stage kidney disease patients on maintenance dialysis are vulnerable to contract COVID-19 infection and variable degrees of disease severity have been reported. SEHA Kidney Care (SKC) is the largest provider of dialysis services in the United Arab Emirates providing dialysis services to 1180 patients, including 80 patients on peritoneal dialysis. Mortality of COVID-19 among patients with ESKD on dialysis is high and ranges from 14 to $31 \%$ in different published series. Not much is known about antibody response to COVID-19 in this group of patients. Patient population demographics in the UAE are dissimilar to many other countries. The objective of this study was to describe the characteristics of patients and clinical staff who developed COVID-19 RT-PCR positivity and assess antibody response to COVID-19.

Methods: We conducted a retrospective analysis to characterize features of COVID-19 in our adult dialysis population. In addition, IgG antibody serology was also performed to assess response to COVID19.

Results: The incidence of COVID-19 infection in our population was $13 \% .81 \%$ of the patients were males, average age was $53.2 \pm 12$ years, and $95 \%$ were on in-center haemodialysis. The majority of patients (68\%) developed mild disease while $13 \%$ required critical care. Combinations of drugs including Hydroxychloroquine, Favipiravir, Lopinavir, Ritonavir, Camostat, Tocilizumab and steroids were used based on local guidelines. The median time to viral clearance was 15 days [IQR 6-26]. Overall mortality in our cohort was $9.2 \%$.

We performed COVID-19 IgG antibody serology in a subset $(n=87)$ of RT-PCR positive patients. Interestingly, 23 (26\%) patients did not develop any antibody response. We successfully implemented a regular screening strategy for patients and staff to identify asymptomatic carriers with an aim to isolate them from the rest of the cohort.

Conclusions: Our study has highlighted an important finding of lack of antibody response in a quarter of patients with ESKD. This may have significant implications for this group of patients while efforts are underway to develop an effective vaccine against COVID-19. With regular screening of all dialysis patients and staff, asymptomatic carriers can be identified and prevent spread of COVID-19 within the dialysis units.

\section{Introduction}

An outbreak of acute pneumonia emerged in Wuhan, China, in December 2019, which was subsequently identified as a novel RNA virus, initially named "severe acute respiratory syndrome coronavirus 2" (SARSCoV-2) (1) and subsequently reclassified by the World Health Organization as coronavirus disease 2019 (COVID-19) (2). 
Clinical presentation of COVID-19 disease is highly variable, from asymptomatic or mild disease (up to $80 \%$ ), to severe with unilateral or bilateral pneumonia (approximately $15 \%$ ). A minority of patients develop severe bilateral pneumonia and respiratory distress that mandates ventilatory support in the intensive care unit (ICU; $3 \%-5 \%)$. In some cases, a severe immune response to the virus can trigger an exuberant inflammatory reaction accompanied by a 'cytokine storm' characterized by the presence of high levels of II-1ß,IL-6, TNF and other inflammatory chemokines like C-C motif chemokine ligands (CCL)-2,3 and 5 (3). Severe inflammatory response includes increasing pneumonia, myocarditis and renal impairment, which may be fatal. Mortality estimates in the general population range from $1.4 \%$ to $8 \%$ (3) (4)(5). Factors associated with a poor prognosis include advanced age, male sex, and previous comorbidity, particularly cardiovascular events, diabetes mellitus, chronic obstructive pulmonary disease, or a history of cancer (6) (7)(8).

Patients with end stage renal disease (ESRD) on haemodialysis (HD) and peritoneal dialysis (PD) may be at higher risk of contracting and dying from COVID-19 due to their relative immune-incompetence.

Uraemia is associated with impaired leucocyte function, and this may change the response to this viral infection(9). In addition, many patients on dialysis have other co-morbidities like diabetes, hypertension and cardiovascular disease which further increases their risk from COVID-19. One of the first series of 5 HD patients with Covid-19 was reported from China in March 2020 (10). Since then, further case series of COVID-19 in ESKD patients have been published with variable mortality rates.

SEHA Kidney Care (SKC), provides dialysis treatment to 1180 patients across multiple sites in the Emirate of Abu Dhabi and is the largest dialysis provider in the UAE. SKC admitted its first patient with COVID-19 in the middle of March 2020. Whilst SKC was proactive in identifying and isolating patients with and without symptoms, and we aimed to protect patients within dialysis units, we found that community transmission of the virus remained a major issue.

Patients with symptoms and with close COVID-19 contacts were immediately isolated from the rest of the HD population, until their RT-PCR results were available. In addition to providing PPE to patients and staff we undertook screening of all patients and staff.

The objective of this study was to describe the characteristics of patients on dialysis and clinical staff who developed COVID-19 RT-PCR positivity and to assess antibody response to COVID-19 in a subset of patients.

\section{Materials And Methods}

\section{Study design and participants}

Data was collected retrospectively between $1^{\text {st }}$ of March until the $1^{\text {st }}$ of July 2020 , from all SKC units. Nasal swab RT-PCR testing of symptomatic patients and staff was performed as well as carrying out screening in all HD and PD patients and staff to identify asymptomatic patients. 
Study participants included all adult patients on maintenance dialysis in SKC units. No Paediatric patients (<18 years) developed COVID-19 disease or screened positive for the disease and patients with CKD not on renal replacement therapy, renal transplant recipients and patients with acute kidney injury requiring dialysis were excluded.

\section{RT-PCR and Antibody testing}

COVID-19 RT-PCR were performed using Cobas SARS-CoV 2 Test by Roche Diagnostics (Roche Molecular Systems, Branchburg, NJ, USA). From the COVID-19 PCR positive patients, a subset underwent antibody testing for COVID-19 IgG antibodies, and testing was performed using the Abbott Architect SARS-CoV-2 IgG (Abbott SARS-CoV-2 IgG, Abbott Diagnostics, Abbott Park, IL, USA) which detects anti-nucleocapsid protein (11). These and other diagnostics tests had regular and routine quality control as expected within an accredited pathology network.

Institutional review board including an ethics and scientific committee approval was obtained for the study.

\section{Data collection:}

Data were extracted from local data and electronic medical records (Cerner Millennium) and all data were reviewed and checked by the primary investigator and the data collection team. Risk and severity assessments were done by the admitting physician based on defined clinical and chest CT scan results criteria. All data were collected at the time of the admission.

\section{Statistical Analysis}

Continuous variables are expressed as mean (standard deviation) or medians (with interquartile range). Normality was assessed by Shapiro-Wilke, D’Agostino \& Pearson and Kolmogorov-Smirnov tests. Categorical variables are presented as total numbers (percentage) as a contingency table. which were analysed by chi-squared test for categorical variables, with Fischer's exact test used when numbers were low. For statistical analyses, a 2-tailed unpaired t-test was used for parametric continuous variables. The association between variables was tested by Spearman rank co-coefficients. Logistic regression was used to determine odds ratios, and only significant univariate correlates were included in multivariate models. A P-value (alpha) of 0.05 or less was considered significant. Tests were performed using GraphPad Prism version 8.4.3 for Mac, GraphPad Software, San Diego, California USA, www.graphpad.com.

\section{Results}

During the study period 152 patients developed a positive COVID RT-PCR test, which represented $~ 13 \%$ of patients being dialysed in SKC units, most of whom (81\%) were male and mostly dialysing in-central dialysis facilities (95\%), with $5 \%$ on PD at home. There were over 16 different nationalities represented in the group. The average age of COVID-19 PCR positive patients was 52 (SD 12) years, with range of 20-85 
years. Around two thirds (64\%) of patients had symptoms at the time of RT-PCR testing. Contact tracing was performed in all patients, and a COVID-19 positive close contact was identified in $~ 52 \%(79 / 152)$ of patients, suggesting the most likely acquisition route was in the community. In $36 \%$ (56/152), we could not connect the patient to any positive COVID-19 contacts within the dialysis unit (they did not dialyse in the same bay/shift or travel with any known positive Covid-19 patient), suggesting that they also likely to have contracted the virus outside the dialysis unit. We found $5 \%(7 / 152)$ who we traced to a possible contact within the dialysis unit and where we may have had a dialysis unit transmission event, as they dialysed in proximity to an index case within the unit (same bay/shift). A further $4 \%(6 / 152)$ cases were linked to a positive case by shared transport to the dialysis unit before we changed transport options for patients. A final 3\% (4/152) appeared to have acquired during hospital admissions due to close contacts with positive cases in hospital.

Those patients whose presenting symptoms were recorded in the notes, reported fever and cough as the most common complaints ( $49 \%$ and $48 \%$ respectively). Other symptoms included dyspnoea (16\%), myalgias (13\%), gastrointestinal symptoms (6\%) and loss of taste $(1.3 \%)$ were reported in our patients. Significant associations with outcome are given in table 1. Most frequent comorbidities were hypertension (93\%), diabetes (49\%) and ischemic heart disease (30\%). Risk assessment was performed at admission and patients were categorized into mild (68\%), moderate $(21 \%)$ and severe $(11 \%)$ disease, based on clinical, laboratory and chest imaging findings. Around half the patients had an abnormal chest $\mathrm{X}$ ray $(45 \%)$ or CT $(52 \%)$.

Table 1: Clinical features of the dialysis patients who had positive COVID-PCR 


\begin{tabular}{|c|c|c|c|c|}
\hline & $\begin{array}{l}\text { Total } \\
(n=152)\end{array}$ & $\begin{array}{l}\text { Alive } \\
(n=138)\end{array}$ & $\begin{array}{l}\text { Died } \\
(n=14)\end{array}$ & P-value* \\
\hline \multicolumn{5}{|l|}{ Demographics } \\
\hline Age, y & $52.4(12.1)$ & $51.2(11.3)$ & $64.1(3.5)$ & 0.002 \\
\hline Male Sex, \% & $81(123)$ & $81(112)$ & $79(11)$ & ns \\
\hline \multicolumn{5}{|l|}{ Co-morbidities } \\
\hline Diabetes, \% (n) & $51(78)$ & $54(75)$ & $21(3)$ & 0.024 \\
\hline Cardiovascular disease, \% (n) & 70 (106) & $72(100)$ & $43(6)$ & 0.032 \\
\hline Smoker, \% (n) & $43(65)$ & $41(57)$ & $57(8)$ & ns \\
\hline \multicolumn{5}{|l|}{ Presenting symptoms } \\
\hline Fever, \% (n) & $49(74)$ & $44(61)$ & $93(13)$ & $<0.001$ \\
\hline Cough, \% (n) & $47(72)$ & $43(60)$ & $86(12)$ & 0.004 \\
\hline Dyspnoea, \% (n) & $16(24)$ & $12(16)$ & $57(8)$ & $<0.001$ \\
\hline Myalgia, \% (n) & $13(19)$ & $12(17)$ & $14(2)$ & ns \\
\hline GI symptoms, \% (n) & $6(9)$ & $4(6)$ & $21(3)$ & 0.038 \\
\hline \multicolumn{5}{|l|}{ Disease severity } \\
\hline Mild, \% (n) & $68(104)$ & $75(103)$ & $7(1)$ & \\
\hline Moderate, \% (n) & $21(32)$ & $20(28)$ & $29(4)$ & \\
\hline Severe, \% (n) & $11(16)$ & $44(7)$ & $64(9)$ & $<0.001$ \\
\hline \multicolumn{5}{|l|}{ Radiological findings } \\
\hline Abnormal chest x-ray, \% (n) & $45(68)$ & $40(55)$ & $93(13)$ & $<0.001$ \\
\hline Abnormal chest CT, \% (n) & $53(80)$ & $49(67)$ & $93(13)$ & 0.001 \\
\hline
\end{tabular}

*P-value determined using two-sample Wilcoxon rank-sum (Mann-Whitney) test for continuous variables and Fisher's exact test for categorical variables. 
Different pharmacological combinations were used as recommended in the UAE national guideline and defined by disease severity, but treating physicians had flexibility to choose different drug regimens from the guidelines. Details of drugs used in mild, moderate and severe disease are given in figure 1. Associations of treatment and outcome are given are in table 2. All patients received prophylactic anticoagulation unless absolutely contraindicated.

Table 2: Drug treatments in patients who were treated for COVID

\begin{tabular}{|lllll|}
\hline Drugs & Total & Alive & Dead & $\mathrm{p}$ - value \\
\hline Hydroxychloroquine or chloroquine & 60 & 50 & 10 & $\mathrm{~ns}$ \\
\hline Favipiravir & 101 & 100 & 1 & $\mathrm{p}=0.28$ \\
\hline Lopinavir and Ritonavir & 13 & 6 & 7 & $\mathrm{~ns}$ \\
\hline Camostat & 48 & 48 & 0 & $\mathrm{~ns}$ \\
\hline Azithromycin & 7 & 4 & 3 & $\mathrm{p}<0.002^{*}$ \\
\hline Steroid treatment & 12 & 12 & 0 & $\mathrm{P}<0.0001^{*}$ \\
\hline Tocilizumab & 8 & 4 & 4 & $\mathrm{p}<0.0001^{*}$ \\
\hline
\end{tabular}

\section{$\mathrm{p}<0.05$ is significant}

ICU admission was required in $13 \%$ of COVID-19 positive patients, $90 \%$ of these needed mechanical ventilation and $70 \%$ required continuous renal replacement therapy (as opposed to continuing with intermittent dialysis therapy). Despite ICU admissions $65 \%$ died. The overall mortality in our population was $9.2 \%$ (14/152), and median time from positive PCR to death in this group was 13 days [IQR 3-29].

Blood group was available in 146 of the 152 patients and blood groups are shown in table 3 . In order to test the association with blood group and infection we compared observed blood group in infected individuals with quoted frequencies for the UAE (12). When blood group 0 was compared with non-O groups, Fischer's exact test yielded $p=0.03$, with an OR $0.59(95 \% \mathrm{Cl} 0.37$ to 0.96$)$, suggesting that blood group 0 may be associated with lower risks of infection. However, when we corrected this calculation for the expected for ethnicity (13), this association was lost (table 3).

Table 3: Distribution of blood groups where known in study population 


\begin{tabular}{|c|c|c|c|c|}
\hline & Quoted (12)(25) & UAE nationals (13) & Ethnically adjusted & Observed Frequency \\
\hline 0 & 48.4 & 56 & 41 & 28.1 \\
\hline A & 24 & 26.2 & 25 & 17.1 \\
\hline B & 22.9 & 14.2 & 27 & 18.5 \\
\hline \multirow[t]{2}{*}{$A B$} & 4.7 & 3.6 & 7 & 4.8 \\
\hline & $p=n s$ & $p=n s$ & $p=n s$ & \\
\hline 0 & 48 & 56 & 41 & 36 \\
\hline non-0 & 51.6 & 44 & 59 & 64 \\
\hline & $p=0.03$ & $p=0.006$ & $p=n s$ & \\
\hline
\end{tabular}

The median time to viral clearance as defined by a first positive to the first of two negative real time polymerase chain reaction (RT-PCR) was 15 days [IQR 6-26] in those that cleared the virus and survived. We noted that occasional patients had subsequently positive PCR result after two negatives, this was rarely more than one positive PCR and we did not count this as a relapse.

A subset of positive patients (87) underwent COVID-19 nucleocapsid IgG antibody testing and most (74\% 64/87) were positive. In patients who were antibody positive the median time to viral clearance was 19 days (IQR 12-30). In contrast those in whom an IgG antibody was not detected had much shorter times from positive to first negative PCR (median 3 days [IQR 2-5]) $p<0.0001$, (figure 2). In patients who were not tested for IgG antibody the median positive time was 15 [IQR 6-29] days which was not significantly different to the $\lg G$ positive patients.

In the COVID-19 PCR positive patients we report no detectable antibodies in $26 \%$ (23/87) of those tested. The majority of these (78\%) were asymptomatic (18/23) and most had a single positive PCR test before two negatives, with a median number of positive PCRs in this group was 1 (range1-2) and we cannot exclude these as false positive PCR tests (representing $11 \%$ of all patients). 5 other interesting patients were those in whom the clinical presentations were consistent with COVID-19 disease and with a positive PCR test, but in whom antibodies did not develop. In this group 3 had a single positive PCR tests, but in two patients had 5 and 8 positive PCR results respectively making it highly probable they did indeed have COVID-19 infection but did not develop antibodies.

All staff were regularly screened and also tested if they were symptomatic, with $24(\sim 10 \%)$ clinical staff testing positive for COVID-19 PCR tests. Of these 63\% (15/24) were symptomatic, but thankfully all staff recovered. Symptomatic staff had longer times 13 [IQR 10-22] days, from positive to two negative tests, compared with asymptomatic staff whose time to negativity was 2 [IQR0-8] days, $p<0.001$. We were 
interested in the asymptomatic group 38\% (9/24). We do not know if these are false positive tests or viral acquisition and rapid clearance but they had a single positive test followed by two negative ones all within usually $96 \mathrm{hrs}$. All positive staff were immediately isolated, reviewed in hospital, and did not return to duty for at least 14 days after their second negative PCR. Many $46 \%(11 / 24)$ of the total positive clinical positive staff had close COVID contacts outside work which are likely to have been the cause of their acquisition of the virus or possibly the false positive test. In the rest (13) we cannot exclude a failure of PPE precautions, although 4 of these were in the asymptomatic probable false positive category, making the maximum number of likely transmission events to be $\sim 9$ if there was no community transmission in the study period.

\section{Discussion}

During COVID-19 pandemic in the UAE, the vulnerability of the dialysis population was of concern. From early in the disease we instituted a number of protective strategies (ref to international guideline to protect patients) to keep patients and staff safe. These included, but were not limited to, providing personal protective equipment (PPE) to all patients, reducing accompanying relatives, social distancing, temperature monitoring with thermal cameras and symptoms questionnaire on arrival, isolation of patients with symptoms or a fever. A strength of this study was the fact that we screened all patients regularly every 2 weeks during the peak of the outbreak in the UAE, as was part of the national strategy. UAE has the some of the highest rates of screening tests performed per million population, in the world to identify asymptomatic patients with COVID-19, at the peak of pandemic. This means that we found a number of our positive patients were asymptomatic, and it means that we are relatively confident that we have captured most of the positive patients. We also found a particularly high proportion of RT- PCR tests that could have been false positive tests, both within staff and patients, but the significance of these findings is unknown. Whilst we very definitely had patients within our facilities who were no doubt excreting virus, and we were very careful to ensure that we protected our staff and patients as far as we could with PPE we would have liked to have followed the very briefly positive staff with antibody testing and this has not been done and is a missed opportunity.

Another strength was the detailed contact tracing that occurred in our patients suggesting that those who acquired the virus mainly did so from contacts outside the dialysis units, suggesting that the strategies we had for protecting our patients mainly worked within the confines of our dialysis facilities.

Furthermore, all COVID positive dialysis patients were assessed in hospital with blood tests and at least a CXR. On discharge we dialyzed any COVID positive patients in isolation with level 3 PPE for 14 days after the last negative. We also cohorted our patients based on a risk assessment of their living conditions outside dialysis to try to keep patients as safe as possible. Those with high density communal living were considered highest risk and PD patients were instructed on self-isolation. We were assisted in our COVID response by the support of the Abu Dhabi Dept of Health who made funding available, and the SEHA procurement, distribution and emergency coordination teams who ensured that we had no PPE shortages, and had dialysis facilities with enough isolation rooms. 
Despite the community transmission, it appears we were relatively successful in protecting most of our patients (and staff) from COVID-19, with a lower percentage of patients within our centres developing COVID 19 compared with international comparators. The overall mortality in our population was $9.2 \%$. Some series have mortality rates of $14-31 \%$ in China (14), United Kingdom 19\% (15), Turkey $20 \%$ (16), Spain 23\% (5), Italy $28 \%$ (17), and the United States 31\% (18). A recent published study from French dialysis units (COVIDIAL) reported mortality of $24 \%$. Median age in this population was 77 (IQR 68-83) years and in whose who died was 80 (IQR 72-88) years. $66 \%$ of those who died in this study were above the age of 75 years (19). The relatively low mortality within our cohort may reflect a relatively young median age of dialysis patient and older age definitely appears a risk factor for mortality, as is clear from the associations with mortality (tables 1\&2). The associations of mortality with age appears relatively robust and may explain some of the relatively low death rate reported here.

There is a univariate association with smoking. Symptoms predicting mortality were dyspnoea and Gastrointestinal symptoms. The association of severe disease and CT changes are unsurprising but the inverse association of comorbidity (diabetes and cardiovascular disease) may well be a type 1 error, related to low numbers. The COVIDIAL study also reported higher mortality in patients who presented with dyspnoea ( $70 \%$ versus $45 \% ; P=0.027$ ). In patients who died, diabetic nephropathy was more common (48 versus $32 \%$ ) and had a history of ischemic heart disease (61 versus $41 \% \mathrm{p}=0.084$ ), however, none of the specific comorbidities were associated with high risk of death (19)

There is a reported association between blood group and COVID-19 infection, with group A being more susceptible and group 0 relatively protected (20-22). In order to assess whether there was such an association in our data we compared the observed to expected frequency of COVID-19 positive patients was similar to quoted figures for the UAE populations (12-13), we confirmed this association but when corrected for indicative population frequencies, these numbers did not meet statistical significance.

The small numbers treated with antivirals and the number of deaths means that although we were able to statically associate comorbidities and treatment with outcome, we cannot statistically draw much conclusion as to the strength of such an association, nor the direction of causality. For example, although there was statistical difference in survival with those given steroids and tocilizumab, this may simple mean that more of the sicker patients received this therapy compared to those that lived. We also present the statistical data with the caveat the number are low, and conclusions around which treatments were helpful in such patients are difficult to extrapolate.

A novel finding of this study relates to the presence of COVID IgG antibodies suggesting that firstly that most Covid-19 positive patients developed an IgG antibody response which may give us hope that they have now protective immunity at least temporarily. It will be interesting to explore how long lived such antibodies are. Secondly, it suggests that some patients $(n=23)$ did not develop an antibody response (and thus presumably protective immunity) despite apparent infection and this has not to our knowledge yet been reported. Such antibody responses have been detected in ICU patients and non ICU patients with COVID-19 (23). Whilst we suspect most of such patients may have had a false positive PCR, in at least in 
5 patients we had solid evidence of COVID-19 infection irrespective of the PCR test. We plan to re-test these patients, but it does suggest that at least some of our patients do not generate a memory response to COVID disease, which remains a worry, especially since when a COVID-19 vaccine arrives, we are hoping that dialysis patients will develop protective immunity. We are however cognisant of the fact that many vaccines, for example hepatitis $B$ are relatively less effective in the dialysis population (24) than with those without renal impairment, so we are likely to need special seroconversion studies in this population.

\section{Conclusion}

Patients with ESKD on dialysis are at high risk of contracting COVID-19 virus infection. The epidemic curve seems to be on a downward trend in many countries, including the UAE, however, there is a concern of a second wave. Our study has indicated an important finding of failure to generate a detectable antibody response in a number of patients. The significance of this finding may be relevant when an effective vaccine against COVID-19 is developed as seroconversion levels in this group may be lower. We have also shown that community transmission of COVID-19 remains the largest threat to our patients and staff, when an effective screening strategy, infection prevention and control measures are instituted within dialysis units.

\section{Declarations}

- All methods were carried out in accordance with relevant guidelines and regulations (Declaration of Helsinki).

- Institutional review board including an ethics and scientific committee approval was obtained from the department of health, Abu Dhabi Health COVID19 Research Ethics Committee.

- Waiver to consent was approved by the department of health Abu Dhabi Health COVID19 Research Ethics Committee. It was requested as it is a retrospective medical record review and study will not have any impact on patient management.

- All authors give consent for the publication of this manuscript

- The datasets used and/or analyzed during the current study are available from the corresponding author on reasonable request.

- The authors declare that they have no competing interests.

- The authors declare that they have not received any funding for this research.

- Authors contributions.

Wasim Ahmed: Conceptualization, writing proposal, data curation, Writing original draft

Ali Abdulkarim Al Obaidli: Methodology, Writing - review \& editing

Princy Joseph: data curation, project management, infection prevention and control 
Edward Smith: Formal analysis, Writing- review \& editing

Ayaz Ahmad Khan: Methodology, data curation, writing- review \& editing

Siddiq Anwar: Methodology, Proposal review, writing- review \& editing

Thangavelu Chandrasekar: Methodology, data curation, writing- review \& editing

Ayman Kamal Al Madani: Proposal review, methodology, Writing- review \& editing

Hormazdiar Dara Dastoor: Methodology, Proposal review, writing- review \& editing

Imran Zahid: Methodology, Writing- review \& editing

Salah Eldin Khalil Issa: Methodology, Writing- review \& editing

Freddie Agbayani Costales: Methodology, Validation, data collection, Writing- review \& editing

Fatima Al Kindi: Methodology, Writing- review \& editing

Yousef Abdul Rahim Boobes: Methodology, Writing- review \& editing

Mohammed Hassan Hassan: Methodology, Writing- review \& editing

Abraham George: Methodology, Writing- review \& editing

Stephen G Holt: Conceptualization, Data curation, Formal analysis, Writing original draft

- Acknowledgements.

We would like to thank the UAE government and the leadership at SEHA for their overwhelming support during this pandemic. We would also like to appreciate and thank the hard work of all the staff at the SKC who worked tirelessly to provide dialysis services during this difficult time.

\section{References}

1. Guan WJ, Ni Z-YY, Hu YYYHY, Liang WH, Ou CQ, He J-XX, et al. Clinical Characteristics of Coronavirus Disease 2019 in China. N Engl J Med. 2020 Apr;382(18):1708-20.

2. He F, Deng Y, Li W. Coronavirus disease 2019: What we know? [Internet]. Vol. 92, Journal of Medical Virology. John Wiley and Sons Inc.; 2020 [cited 2020 Aug 16]. p. 719-25. Available from: https://pubmed.ncbi.nlm.nih.gov/32170865/

3. Ye Q, Wang B, Mao J. The pathogenesis and treatment of the 'Cytokine Storm' in COVID-19. J Infect. 2020 Jun;80(6):607-13.

4. Grasselli G, Zangrillo A, Zanella A, Antonelli M, Cabrini L, Castelli A, et al. Baseline Characteristics and Outcomes of 1591 Patients Infected with SARS-CoV-2 Admitted to ICUs of the Lombardy Region, 
Italy. JAMA - J Am Med Assoc [Internet]. 2020 Apr 28 [cited 2020 Aug 16];323(16):1574-81. Available from: https://jamanetwork.com/

5. Goicoechea M, Sánchez Cámara LA, Macías N, Muñoz de Morales A, Rojas ÁG, Bascuñana A, et al. COVID-19: clinical course and outcomes of 36 hemodialysis patients in Spain. Kidney Int. 2020;98(1):27-34.

6. Madjid M, Safavi-Naeini P, Solomon SD, Vardeny O. Potential Effects of Coronaviruses on the Cardiovascular System: A Review [Internet]. Vol. 5, JAMA Cardiology. American Medical Association; 2020 [cited 2020 Aug 16]. p. 831-40. Available from: https://jamanetwork.com/

7. Wynants L, Van Calster B, Collins GS, Riley RD, Heinze G, Schuit E, et al. Prediction models for diagnosis and prognosis of covid-19: Systematic review and critical appraisal. BMJ [Internet]. 2020 Apr 7 [cited 2020 Aug 16];369:18. Available from: https://www.bmj.com/content/369/bmj.m1328

8. Guo T, Fan Y, Chen M, Wu X, Zhang L, He T, et al. Cardiovascular Implications of Fatal Outcomes of Patients with Coronavirus Disease 2019 (COVID-19). JAMA Cardiol [Internet]. 2020 Jul 1 [cited 2020 Aug 16];5(7):811-8. Available from: https://jamanetwork.com/

9. Zhou J, Li C, Zhao G, Chu H, Wang D, Yan HHN, et al. Human intestinal tract serves as an alternative infection route for Middle East respiratory syndrome coronavirus. Sci Adv [Internet]. 2017 [cited 2020 Aug 16];3(11). Available from: /pmc/articles/PMC5687858/?report=abstract

10. Wang R, Liao C, He H, Hu C, Wei Z, Hong Z, et al. COVID-19 in Hemodialysis Patients: A Report of 5 Cases. Am J Kidney Dis. 2020;

11. Bryan A, Pepper G, Wener MH, Fink SL, Morishima C, Chaudhary A, et al. Performance characteristics of the abbott architect sars-cov-2 igg assay and seroprevalence in Boise, Idaho. J Clin Microbiol. 2020 Aug 1;58(8).

12. Blood type distribution by country. https://en.wikipedia.org/wiki/Blood_type_distribution_by_country

13. Hamad M, Saeed Z, Meqbali A, Al Meqbali S, Zaher MH. Frequency of ABO, Rhesus Phenotypes and Most Probable Genotypes and the Type of RHD Negative Variants among UAE Nationals in Al Ain District Recommended Citation [Internet]. 2015. Available from:

https://scholarworks.uaeu.ac.ae/all_theseshttps://scholarworks.uaeu.ac.ae/all_theses/243

14. Wu J, Li J, Zhu G, Zhang Y, Bi Z, Yu Y, et al. Clinical Features of Maintenance Hemodialysis Patients with 2019 Novel Coronavirus-Infected Pneumonia in Wuhan, China. Clin J Am Soc Nephrol. 2020;CJN.04160320.

15. Home - The Renal Association [Internet]. [cited 2020 Aug 16]. Available from: https://renal.org/

16. Aydin Bahat K, Parmaksiz E, Sert S. The clinical characteristics and course of COVID-19 in hemodialysis patients. Hemodial Int [Internet]. 2020;n/a(n/a). Available from: https://doi.org/10.1111/hdi.12861

17. Alberici F, Delbarba E, Manenti C, Econimo L, Valerio F, Pola A, et al. A report from the Brescia Renal COVID Task Force on the clinical characteristics and short-term outcome of hemodialysis patients with SARS-CoV-2 infection. Kidney Int. 2020;(May). 
18. Valeri AM, Robbins-Juarez SY, Stevens JS, Ahn W, Rao MK, Radhakrishnan J, et al. Presentation and Outcomes of Patients with ESKD and COVID-19. J Am Soc Nephrol. 2020;31(7):1409-15.

19. Keller N, Chantrel F, Krummel T, Bazin-Kara D, Faller AL, Muller C, et al. Impact of first-wave COronaVIrus disease 2019 infection in patients on haemoDIALysis in Alsace: the observational COVIDIAL study. Nephrol Dial Transplant [Internet]. 2020 Aug 1;35(8):1338-411. Available from: https://doi.org/10.1093/ndt/gfaa170

20. Göker H, Aladağ-Karakulak E, Demiroğlu H, Ayaz CM, Büyükaşik Y, İnkaya AC, et al. The effects of blood group types on the risk of COVID-19 infection and its clinical outcome. Turkish J Med Sci. 2020;50(4):679-83.

21. Fan Q, Zhang W, Li B, Li DJ, Zhang J, Zhao F. Association Between ABO Blood Group System and COVID-19 Susceptibility in Wuhan. Front Cell Infect Microbiol. 2020 Jul 21;10.

22. Latz CA, DeCarlo C, Boitano L, Png CYM, Patell R, Conrad MF, et al. Blood type and outcomes in patients with COVID-19. Ann Hematol. 2020;2113-8.

23. Liu L, To KKW, Chan KH, Wong YC, Zhou R, Kwan KY, et al. High neutralizing antibody titer in intensive care unit patients with COVID-19. Emerg Microbes Infect. 2020;1-30.

24. Barraclough KA, Playford EG. Hepatitis B virus infection in hemodialysis populations: Progress toward prevention. Kidney Int. 2010;77(3):177-80.

\section{Figures}




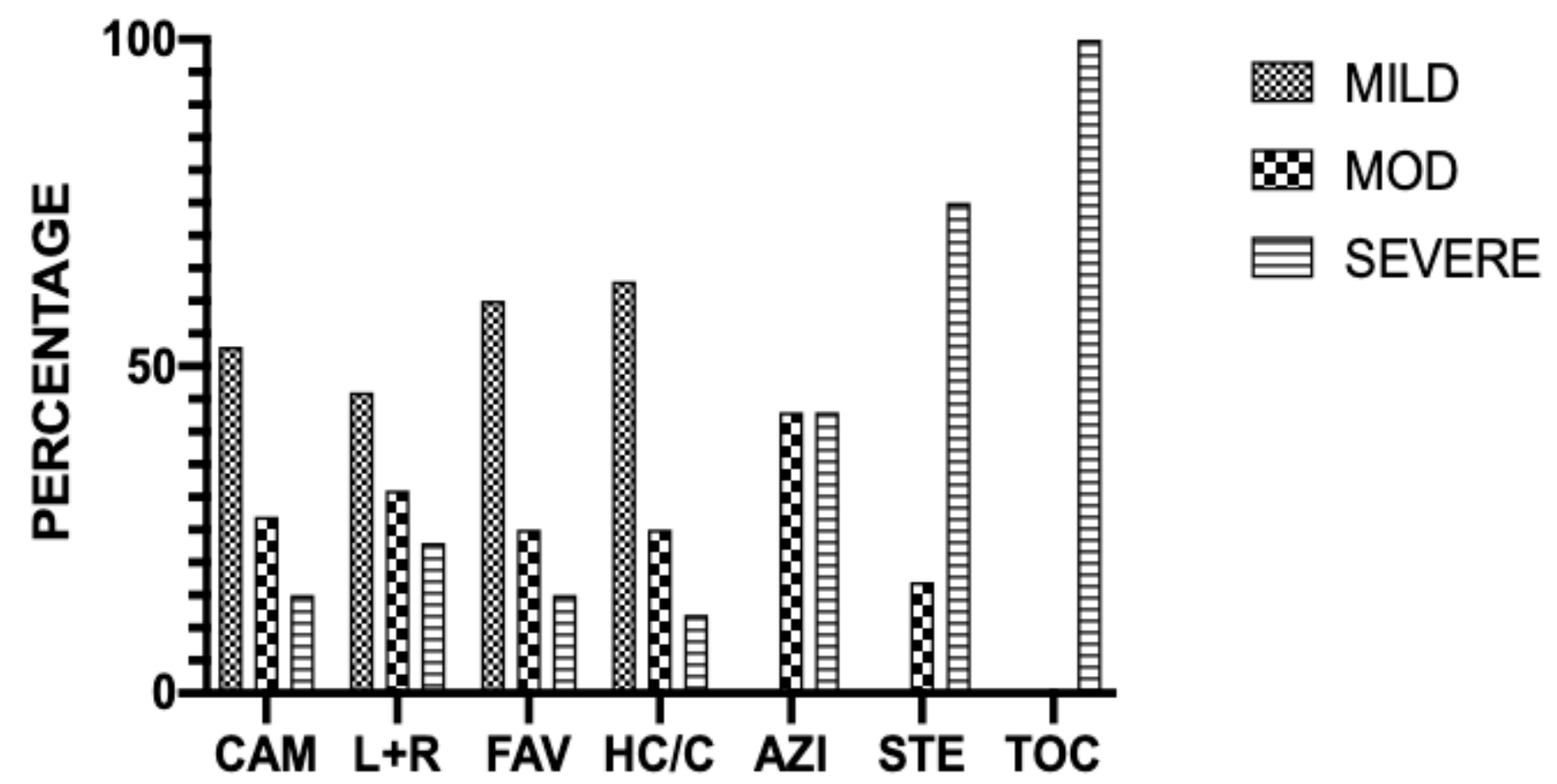

(CAM=Camostat, $\mathrm{L}+\mathrm{R}=$ Lopinavir and ritonavir, $\mathrm{FAV}=$ favipiravir $\mathrm{HC} / \mathrm{C}$ - hydroxychloroquine or

Chloroquine, AZI- Azithromycin, STE= Steroids, TOC = Tocilizumab)

Figure 1

Percent of patients in mild moderate and severe categories receiving drugs therapies 


\section{Time to negative}

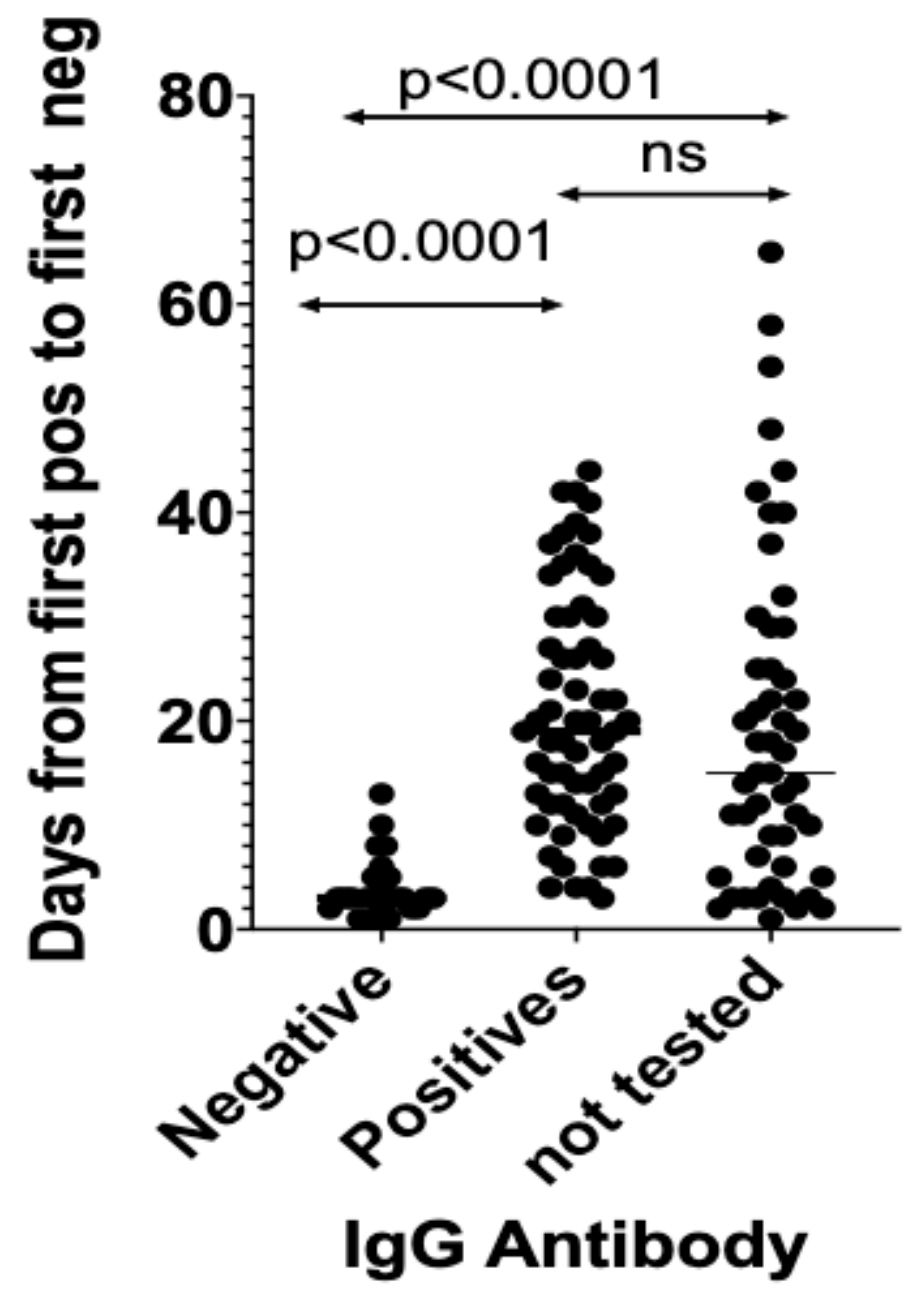

Figure 2

Days from COVID PCR Positive to the first of two negative COVID PCR tests in patients with and without detectable IgG antibodies. There was a highly significant difference in median values in those with antibody results. 